\title{
Multilevel Image Transmission using Polar Codes
}

\author{
Guadalupe Donaji Aguilar Licea, Saul Lazcano Salas \\ Universidad Autónoma del Estado de México, CU Valle de México, Mexico \\ gpe.ag.18@gmail.com, slazcanos@uaemex.mx
}

\begin{abstract}
Nowadays, with the big amounts of data that gets transmitted all the time through the communication systems, and among the different technologies that are in development in order to support future networks, many methods of information processing such as channel coding strategies and multilevel image transmission are being studied in order to be used with the oncoming technologies. One of the most common problem in communication systems, is how intrusive noise inherent to the channel during the transmission can be, as well as the limited bandwidth available, especially in wireless channels. This work presents a multilevel image decomposition using a wavelet transform, and their transmission through an AWGN channel with polar codes as a channel coding strategy, using different frame sizes $(\mathrm{N}=1024, \mathrm{~N}=512$ and $\mathrm{N}=256)$ in order to test how robust polar codes' performance can be for multilevel image decomposition in presence of noise during transmission.
\end{abstract}

Keywords: wavelet, multilevel image decomposition, polar codes, channel coding.

\section{Introduction}

The need to improve communication systems for the exchange of information, has led to different techniques to be created. In terms of $5 \mathrm{G}$ networks, these techniques become paramount, since most of the services expected from it rely on high efficiency and reliability [1]. One of these improvements comes from the work of C. Shannon in 1948, which marks a starting point for the emergence of a new area of work: channel coding, which involves techniques that allow the detection and / or correction of errors that may exist during the transmission process. This can be done in different ways, as long as the encoder and therefore the decoder, are able to identify the errors and correct them afterwards.

Following this line, in 2009, Erdal Arikan made a proposal of a channel coding technique [2], which involves the polarization of the channel, hence calling it Polar Coding. Channel polarization takes the original channel and combines it in order to separate the good channels (noiseless) from bad channels (with high noise) and then, use good channels to transmit the information. This process allows Polar Codes to reach a BER performance close to Shannon's limit using a low complexity coding scheme, making them so attractive to use as a standard channel coding technique for wireless communications, such as $5 \mathrm{G}$ and others.

This paper focuses on analyzing the strengths of Polar Codes in terms of multi -level transmission of wavelet processed images. Wavelets, in image processing, are 
algorithms that make a decomposition of the image and divides it in four different components, each one containing different details. For this analysis, two wavelets were used in order to make a comparison; these wavelets were: Haar [3] because it is the simplest and most common in image processing, making it, in most platforms, the default. The other one was Daubechies 15 [4], due to this being an extension of the Haar wavelet.

The remaining contents of this paper are organized as follows. Section 2 gives some background on wavelets, and a description of polar codes. Section 3 describes the testing scheme for the polar codes. The results of the simulation are shown and described in Section 4. And finally, Section 5 presents the conclusions of the work along with possible future implementation of this study.

\section{Wavelets and Polar Codes}

Wavelets, for being mathematical functions, have been developed in different areas, in image processing they allow to process the information into different frequency components and analyze any discontinuity within the image. Some of these wavelets are derivatives of the Haar and Daubechies transforms. In general, these wavelets decompose the signals in half, having as a result two smaller signals, making it a very simple, fast, and easier to revert.

EZW (Embedded Zerotrees Wavelet) is another algorithm used in image compression [5]. It classifies the transformed coefficients in a tree, where the low frequency coefficients are placed at the root and the higher frequencies get placed at the next subbands of the tree. In [6] an EZW-Polar Code image coding method is proposed in order to help finding more applications for image coding and polar codes.

In other way, as mentioned previously, Polar Codes separate the noisy channels from the noiseless ones, a key step to construct Polar Codes is channel polarization, which is linked to a specific channel parameter (error probability - Pe), in other words, if the Pe changes, polarization changes too, Polar Codes are not universal. In this work, we build Polar Codes according to SNR point selected.

\section{Test Scheme}

The flow chart in Fig. 1 shows the process of the testing system used for both wavelet cases, which includes the transmission system, and the treatment of the image used (Lena 512 X 512). It should be mentioned that the frame lengths used are 1024 bits, 512 bits, and 256 bits, making the channels' lengths 2048, 1024 and 512 respectively.

As it is shown, in order to transmit the image using polar codes, first, it had to be decomposed using the wavelet, which in this case, consisted of a one-level decomposition, see Fig. 2. Afterwards, each resulting image was converted into a vector, so it could be worked with during the simulations.

For all transmissions, an AWGN (Additive White Gaussian Noise) channel was chosen, along with a SNR (Signal to Noise Ratio) that would be just the same as the channel state; in this case, it ranged from 1 to 10 with $\Delta=0.1$. This with the purpose of 
having optimal channel states and SNR at all times and ensure as most as possible, the integrity of the transmitted information. Because the channel is already established, as well as its parameters, it means that the polar codes used for this scheme, are not universal [5].

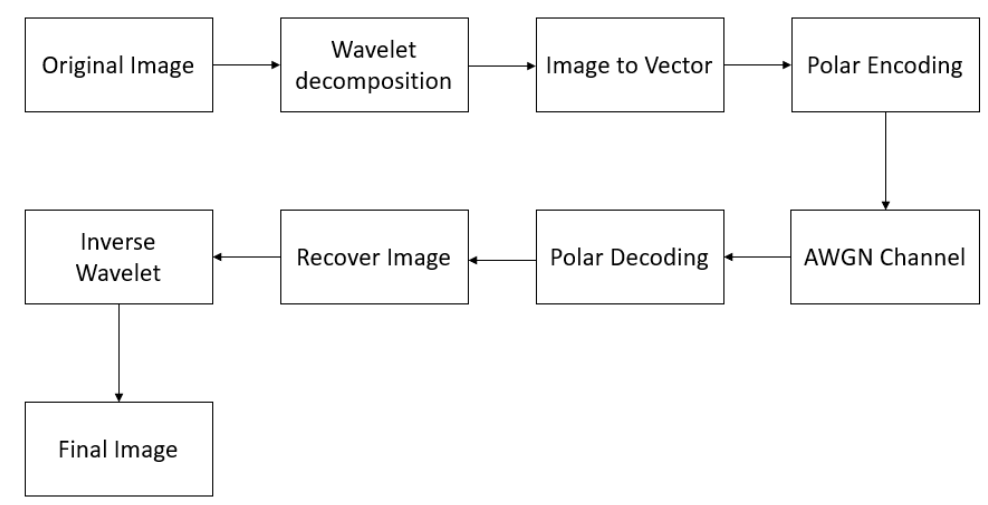

Fig. 1. Transmission system.

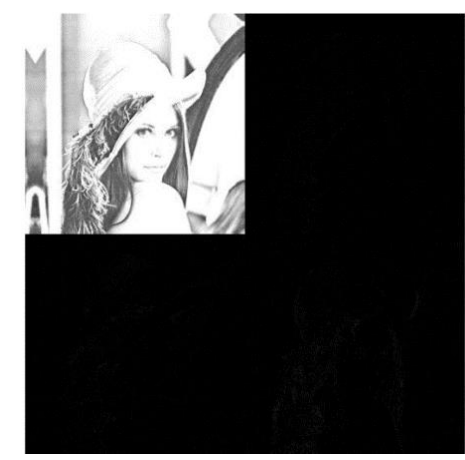

a) DB15

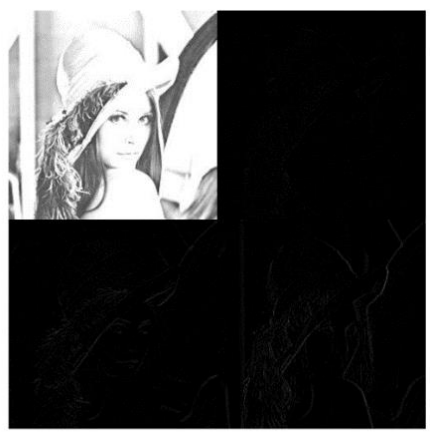

b) Haar

Fig. 2. Images after wavelet composition.

\section{Results}

A comparison of the BER (Bit-Error Rate) and FER (Frame-Error Rate) obtained from the transmission simulation of both wavelets and three frame lengths is illustrated in Fig. 3 to Fig. 5. For the 1024 bits frame, in both wavelet cases, once the SNR reached $+3 \mathrm{~dB}$, the error correction became almost optimal, correcting all errors, with the exception of the DB15 wavelet, where it found 8 BERs and 1 FERs when the SNR channel $=3.8 \mathrm{~dB}$, from then on, the rest of the channel states did not encounter more errors.

A similar situation occurred for $\mathrm{N}=512$, where the error correction became optimal at $+3.4 \mathrm{~dB}$ but for the Haar wavelet, at the SNR channel=3.7dB the BER $=8$ and FER 
$=1$ and again, from then on, the channels became optimal. For $\mathrm{N}=256$, the error correction became optimal until the SNR reached $+4 \mathrm{~dB}$.

Once the transmission simulation was over, the resulting images were recovered by reconstructing the vector into the corresponding image matrix.

The generated errors according to the SNR in the previous graphic, can be appreciated in the images as well. Fig. 6 shows an example of the differences in the errors, where the image sent in the 1024 bits frame has almost half of the errors compared to the resulting ones in both the 512 and 256 bits frame, however none of them have errors once the SNR got higher than $3 \mathrm{~dB}$ and $4 \mathrm{~dB}$ respectively.
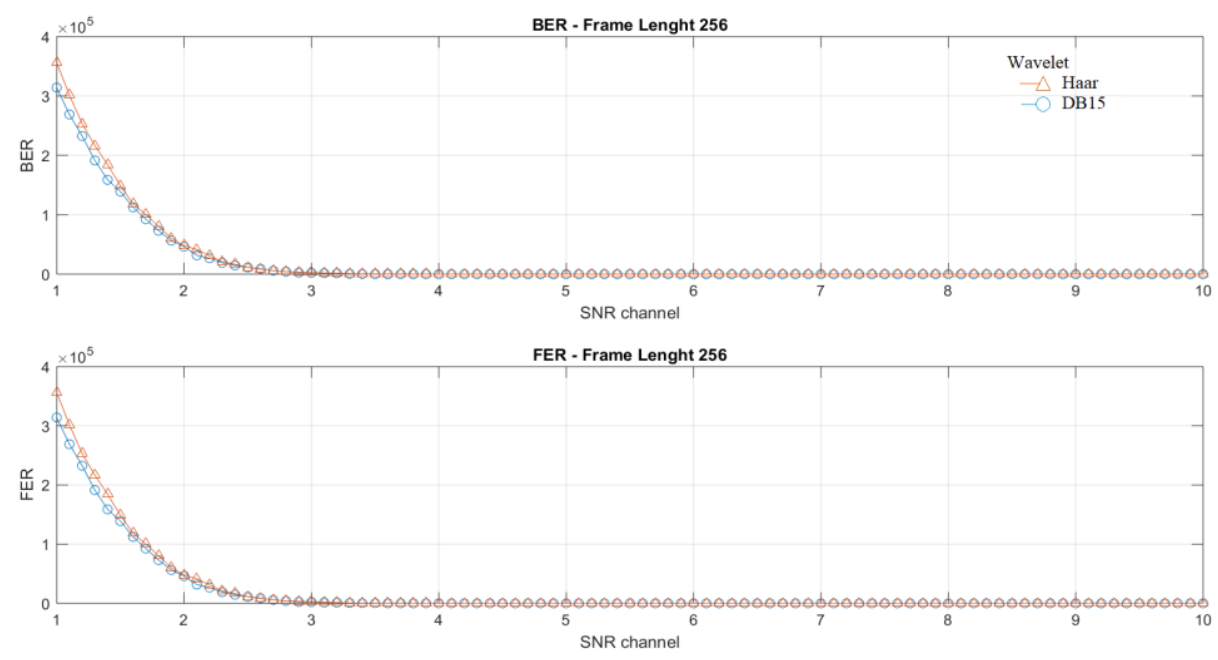

Fig. 3. $B E R / F E R-N=256$ results.
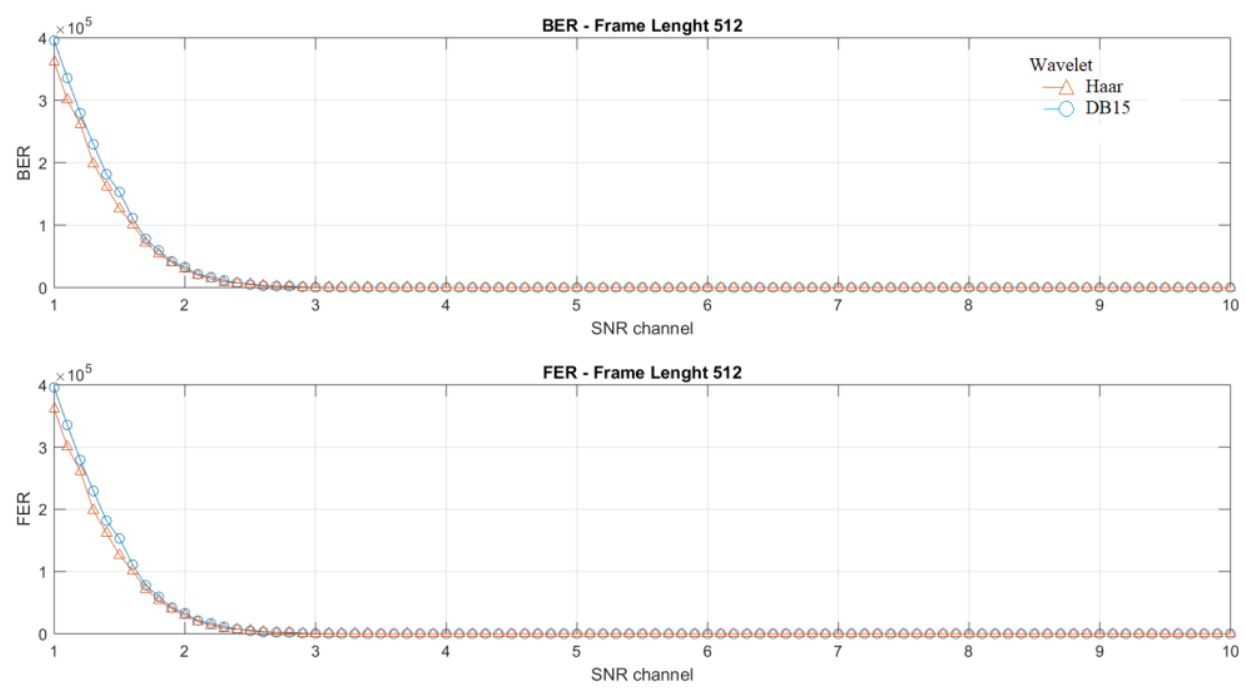

Fig. 4. $\mathrm{BER} / \mathrm{FER}-\mathrm{N}=512$ results. 
Multilevel Image Transmission using Polar Codes
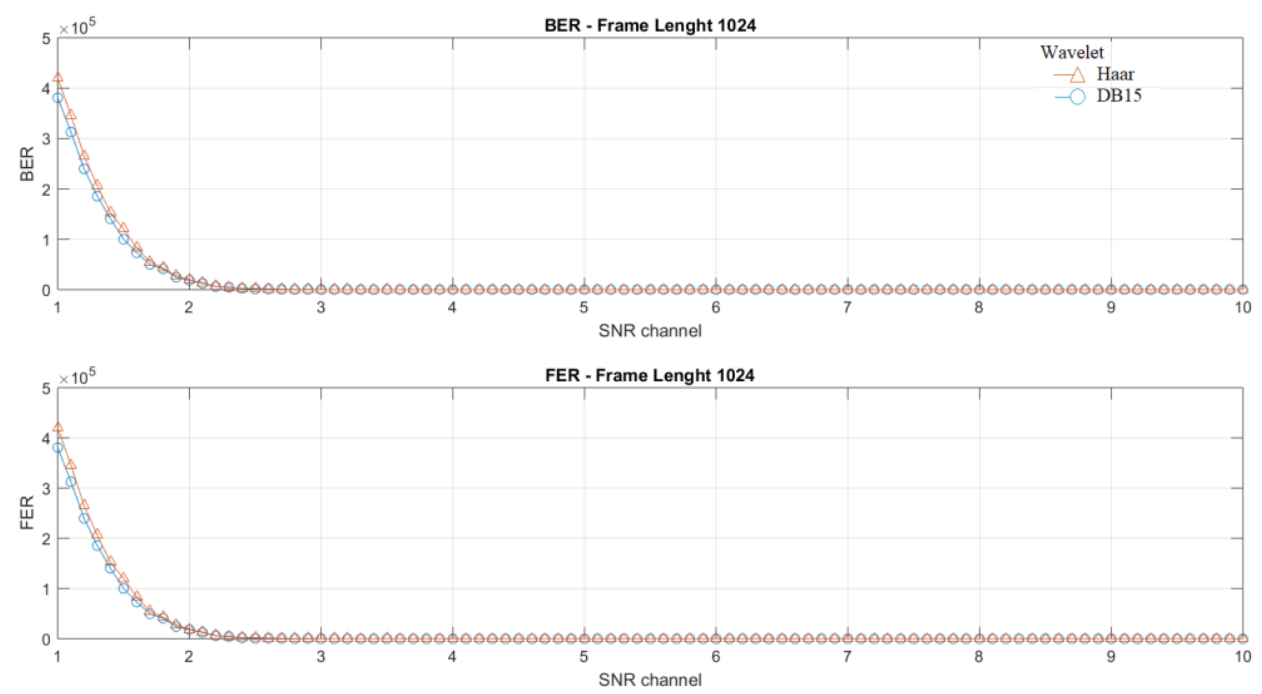

Fig. 5. BER/FER $-\mathrm{N}=1024$ results.
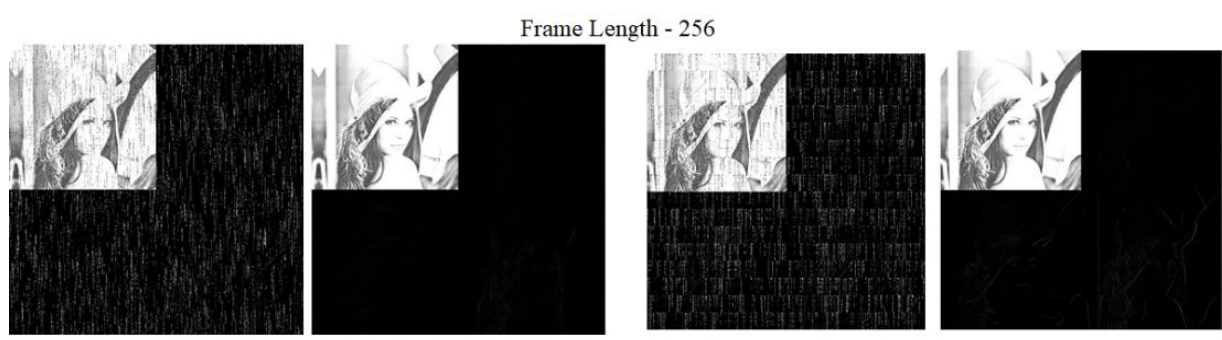

Frame Length -512
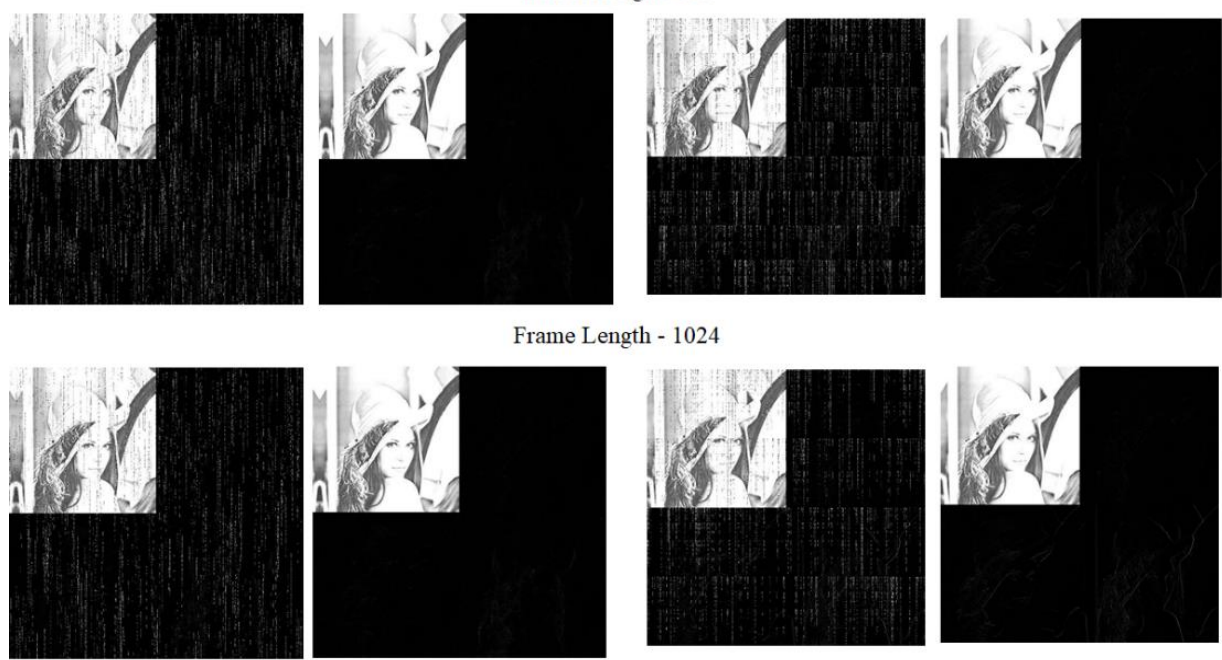

a) $\mathrm{DB} 15$

b) Haar

Fig. 6. Images after transmission with and without errors (SNR: 1.7dB and 9.2dB). 
To make a comparison between the original image and the transmitted ones, it was necessary to make a reconstruction of these obtained images by applying the corresponding inverse wavelet transform to each one. Then three different comparison parameters, such as, MSER (Mean Square Error Rate), PSNR (Peak Signal to Noise Ratio) and SNR (Signal to Noise Ratio) were used in every image along with the original. These results are displayed in Fig. 7 to Fig. 9.

Once the BER and FER got to zero, the resulting images were identical to each other for each wavelet, as well as the original image. For example, the resulted DB15 image from SNR channel at $3.7 \mathrm{~dB}$, is identical to the one obtained from the $9.5 \mathrm{~dB}$ channel, no matter the frame length. The same situation happened with the Haar wavelet obtained images.

Making an analysis of the results in the previous graphics, it became predictable, in all three cases: $\mathrm{N}=256, \mathrm{~N}=512$ and $\mathrm{N}=1024$, that once the BER and FER become zero, the MSER, PSNR and SNR would start giving the same results. For $\mathrm{N}=256$, with the DB15 wavelet, this started in the SNR channel=4.0dB; for the Haar case, it started in the SNR channel $=4.3 \mathrm{~dB}$. When $\mathrm{N}=512$, this happened at the $\mathrm{SNR}$ channel $=3.5 \mathrm{~dB}$ except for the Haar wavelet, where in channel $3.7 \mathrm{~dB}$, the $\mathrm{BER}=8$ and $\mathrm{FER}=1$.

Finally, for $\mathrm{N}=1024$, this situation was presented earlier, for Haar it started in the $3.1 \mathrm{~dB}$ channel, and for DB15 in 3.3db channel, with the exception on channel 3.5, where the $\mathrm{FER}=1$ and $\mathrm{BER}=8$.

These results exhibit a particular behavior of polar codes in terms of performance versus frame lengths, where the smaller the frame the worse the performance, whereas the longer the frame the better the performance gets. [6]
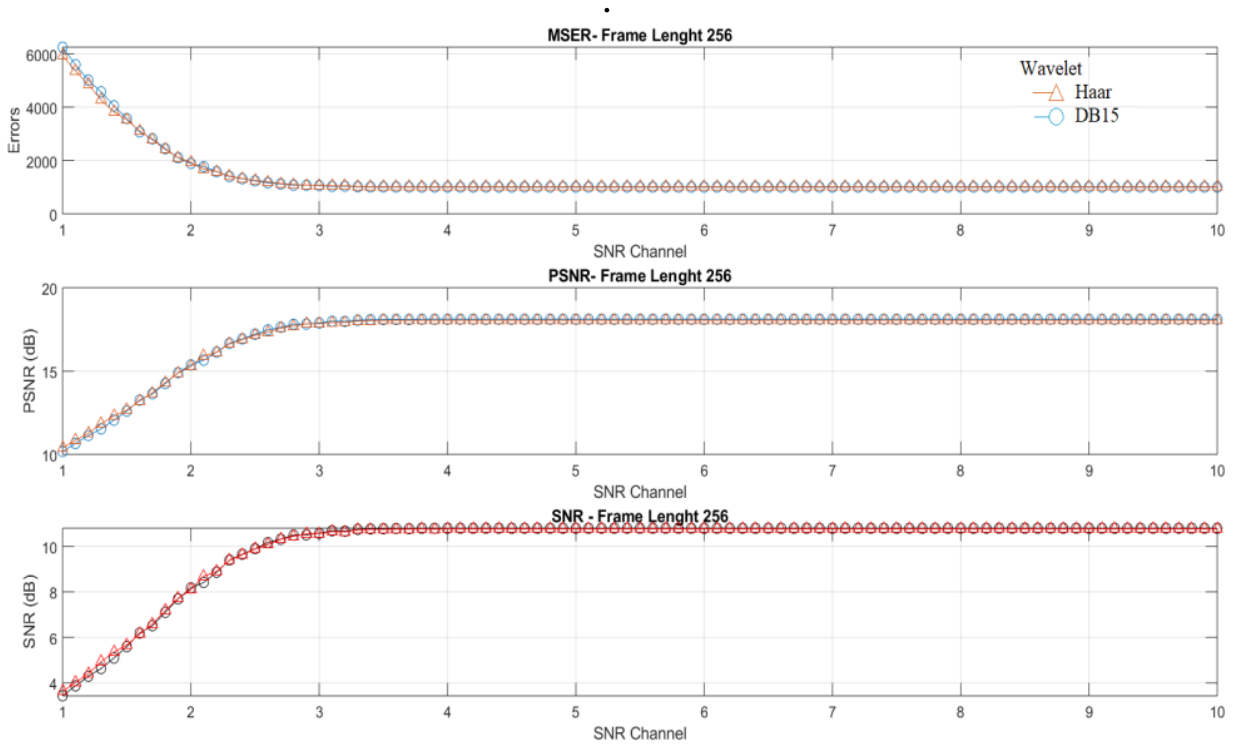

Fig. 7. Image comparison results $\mathrm{N}=256$ 

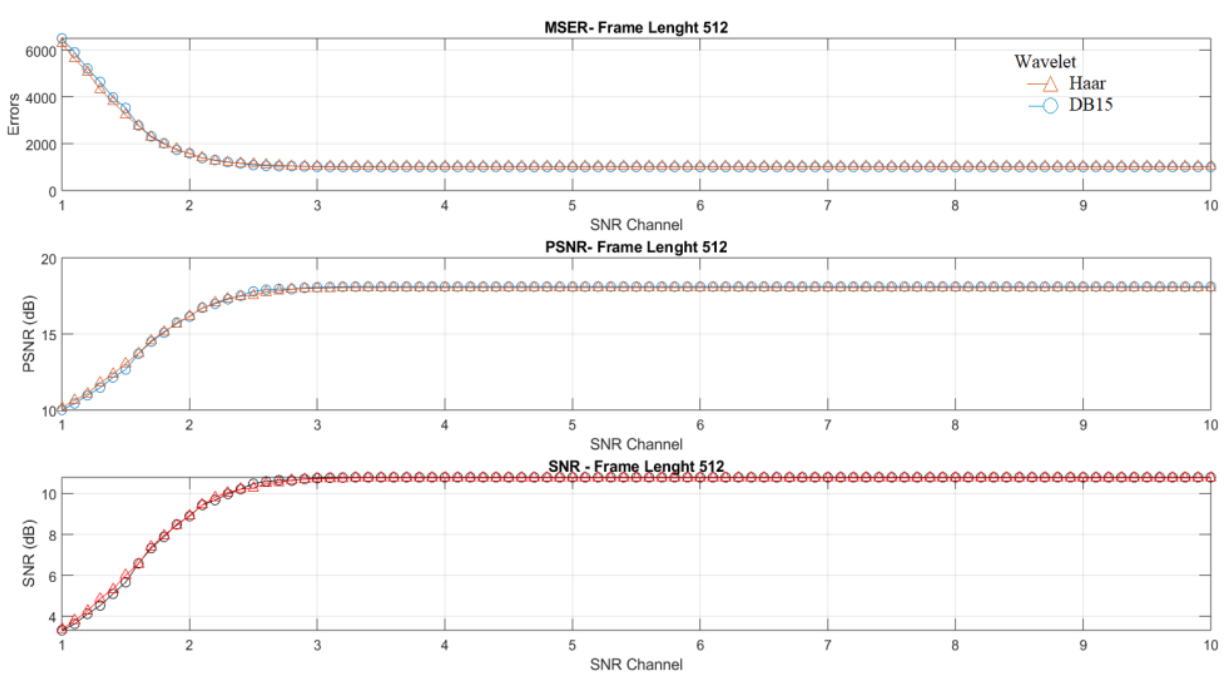

Fig. 8. Image comparison results $\mathrm{N}=512$
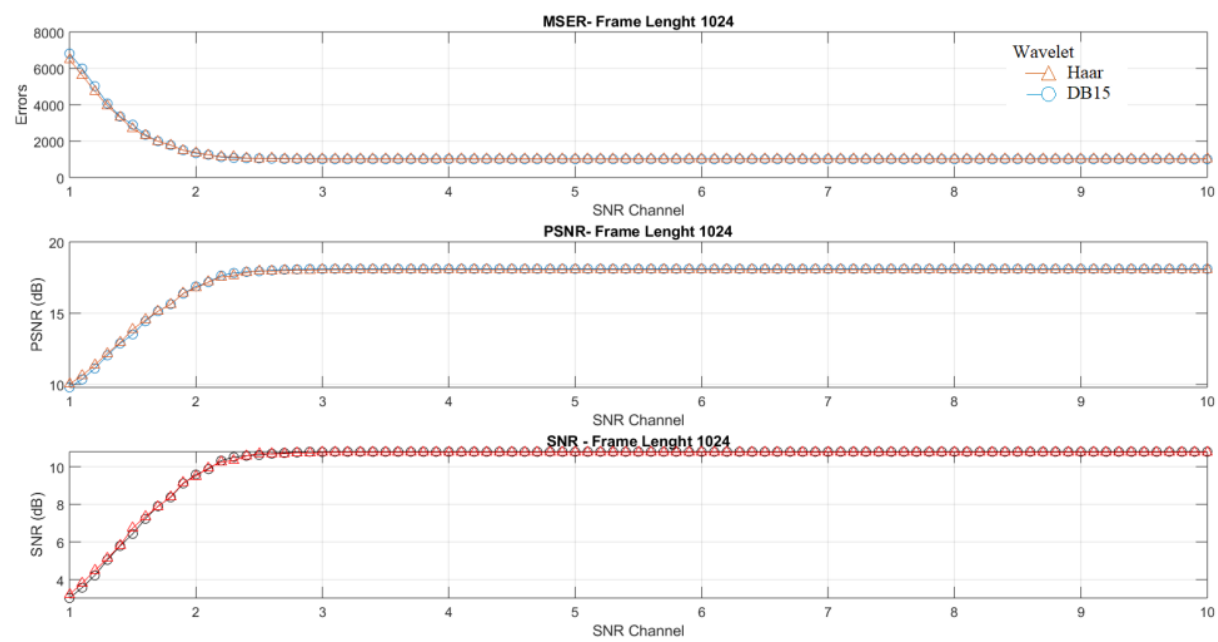

Fig. 9. Image comparison results $\mathrm{N}=1024$

\section{$5 \quad$ Conclusions and Future Work}

It can be concluded that since polar codes have better performance with long frames of information opposed to small ones, such as the ones used nowadays (256 bits), this coding technique is a strong option for $5 \mathrm{G}$, due to the fact that more information can be transmitted at once, and the optimal channel SNR can be reached more easily during the transmission, meaning that the integrity of the information can be maintained as if it were sent in smaller frames.

This test only included a single level decomposition, but hopefully it can open the door to future studies that could involve the use of the frozen bits or frozen channels, 
where the decomposition of the image via wavelets play a more active role, meaning that the wavelets would involve a greater level of decomposition and therefore, the main component of the image is sent through the optimal channels and the rest of the details are sent through the less optimal channels, making the transmission a bit faster by lowering the redundancy, and ensuring that at least the main component will be received without or at most, with minimal errors.

Following this line of transmissions using polar codes, this technique can be implemented in SDR (Software Defined Radio) devices, as well as platforms, being GNU Radio the main platform because of the extensive utilities it offers, and since it is open source, the user can create as many tools as necessary.

\section{References}

1. ITU: Document 5D/TEMP/300 (Rev.1)

2. Arikan, E.: Channel polarization: A method for constructing capacity-achieving codes. In: IEEE International Symposium on Information Theory (2008)

3. Haar, A., Zimmermann, G.: On the Theory of Orthogonal Function Systems. Fundamental Papers in Wavelet Theory, pp. 155-188. doi:10.1515/9781400827268.155 (2009)

4. Daubechies, I.: Orthonormal bases of compactly supported wavelets. Communications on Pure and Applied Mathematics 41(7), 909-996. doi:10.1002/cpa.3160410705 (1988)

5. Shapiro, J.M.: Embedded image coding using zerotrees of wavelet coefficients. IEEE Transactions on Signal Processing 41(12), 3445-3462 (1993)

6. Ma, H.M., Chen, D., Zhang, C.: Embedded zerotrees wavelet image coding using source polar codes. In: 2017 IEEE 17th International Conference on Communication Technology (ICCT). doi:10.1109/icct.2017.8359927 (2017)

7. Hassani, S.H., Urbanke, R.: Universal polar codes. In: 2014 IEEE International Symposium on Information Theory. doi:10.1109/isit.2014.6875073 (2014)

8. Payommai, T., Chamnongthai, K.: Performance of polar code for image transmission. In: 2013 International Symposium on Intelligent Signal Processing and Communication Systems. doi:10.1109/ispacs.2013.6704592 (2013) 